\title{
Dominated and Bounded Convergence Results of Sequential Henstock Stieltjes Integral in Real Valued Space
}

\author{
V. O. Iluebe ${ }^{1}$, A. A. Mogbademu ${ }^{2}$ \\ 1 victorodalochi1960@gmail.com, Department of Mathematics, University of Lagos, Akoka \\ 2 amogbademu@unilag.edu.ng, Department of Mathematics, University of Lagos, Akoka \\ Correspondence to: V. O. Iluebe, Email: victorodalochi1960@gmail.com
}

\begin{abstract}
In this paper, we prove the dominated and bounded convergence results for real-valued Sequential Henstock Stieltjes integral.
\end{abstract}

Keywords: Sequential Henstock Stieltjes integrable, Increasing functions, Guages, Dominated and bounded convergence, Uniform-integrability

DOI: https://doi.org/10.3126/jnms.v3i1.32999

\section{Introduction and Preliminaries}

In 1955 and 1957 respectively, R. Henstock and J. Kursweil independently gave a Riemann-type integral called the Henstock integral. It is a kind of non-absolute integral which includes the Riemann, Improper Riemann, Newton and Lebesgue integral. Many authors have studied Henstock integral which is now known as the Kursweil-Henstock integral, since Kursweil defined the same integral though they went different ways in developing and applying the theory. For simplicity, we shall refer the Kursweil-Henstock integral and its general form as the Henstock integral in this paper, see [1]-[6]. It is well known that the Henstock integral is equivalent to the Denjoy integral, Perron integral and Denjoy-Perron integrals. The equivalence of the Henstock integral and Sequential Henstock integral has been discussed in [5]. In this paper, we prove the dominated and bounded convergence theorems for the Sequential Henstock Stieltjes integral.

The symbols used in this paper are as follows: $\mathbb{R}$ and $\mathbb{N}$ for a set of real and natural numbers respectively, $\left\{\delta_{n}(x)\right\}_{n=1}^{\infty}$ as set of gauge functions of $x \in[a, b]$, and $P_{n}$ as set of partitions of subintervals of a compact interval $[a, b]$ for $n=1,2,3, \cdots$

The following useful definitions of Sequential Henstock integral are needed.

Definition 1.1. [5](Sequential Henstock Integral). A function $f:[a, b] \rightarrow \mathbb{R}$ is Sequential Henstock integrable on $[a, b]$ if there exists a number $\alpha \in \mathbb{R}$ and a sequence of gauge functions $\delta_{\mu}(x) \in\left\{\delta_{n}(x)\right\}_{n=1}^{\infty}$ such that for each $\delta_{n}(x)$-fine tagged partitions $P_{n}=\left\{\left(u_{(i-1)_{n}}, u_{i_{n}}\right), t_{i_{n}}\right\}$ we have

$$
U\left(f, P_{n}\right)=\sum_{i=1}^{n \in \mathbb{N}} f\left(t_{i_{n}}\right)\left(u_{i_{n}}-u_{(i-1)_{n}}\right) \rightarrow \alpha, n \rightarrow \infty
$$

i.e. $\alpha=\int_{[a, b]} f$.

Sequel to Definition 1.1, we give a new definition as follows for Sequential Henstock Stieltjes integral.

Definition 1.2. (Sequential Henstock Stieltjes Integral). A function $f:[a, b] \rightarrow \mathbb{R}$ is Sequential Henstock Stieltjes integrable on $[a, b]$ with respect to an increasing function $g:[a, b] \rightarrow \mathbb{R}$ if there exists a number $\alpha \in \mathbb{R}$ such that for $\varepsilon>0$ there exists a sequence of positive functions $\delta_{\mu}(x) \in\left\{\delta_{n}(x)\right\}_{n=1}^{\infty}$ such that $\mu \leq n$ and for every $\delta_{n}(x)$-fine tagged partitions $\left.P_{n}=\left\{\left(u_{(i-1)_{n}}\right), u_{i_{n}}\right), t_{i_{n}}\right\}$ and $u_{(i-1)_{n}} \leq t_{i_{n}} \leq u_{i_{n}}$ we have

$$
\left.U\left(f, g, P_{n}\right)=\sum_{i=1}^{n} f\left(t_{i_{n}}\right)\left[g\left(u_{i_{n}}\right)-g\left(u_{(i-1)_{n}}\right)\right)\right] \rightarrow \alpha, \text { as } n \rightarrow \infty .
$$


We say that $\alpha \in \mathbb{R}$ is the Sequential Henstock Steiltjes integral of $f$ with respect to $g$ on $[a, b]$ with $\alpha=\int_{a}^{b} f d g$

Definition 1.3. [5] (Uniform Integrability). Let $f_{k}:[a, b] \rightarrow \mathbb{R}$ be a sequence of functions for $k \in \mathbb{N}$ and a function $g:[a, b] \rightarrow \mathbb{R}$. Then $f_{k}$ is uniformly Sequential Henstock Stieltjes integrable with respect to $g$ on $[a, b]$ if

i. the integral $\int_{a}^{b} f_{k} d g$ exists for each $k \in \mathbb{N}$,

ii. for $\varepsilon>0$ there exists a sequence of gauges $\delta_{\mu}(x)=\sup _{\mu \in \mathbb{N}}\left\{\delta_{n}(x)\right\}_{n}^{\infty}$ and $n \geq \mathbb{N}$ on $[a, b]$ such that the inequality

$$
\left|\int_{a}^{b} f_{k} d g-U\left(f_{k}, d g, P_{n}\right)\right|<\varepsilon,
$$

holds for each $\delta_{n}(x)$ - fine partition $P_{n}$ of $[a, b]$ for $n=1,2,3, \cdots$.

Now, we state the following lemma which was proved in 4, and useful in the proof of our main theorems.

Lemma 1.1. 4] Let $f_{n}:[a, b] \rightarrow \mathbb{R}$ be a sequence of functions and $g:[a, b] \rightarrow \mathbb{R}$ be an increasing function satisfying the following conditions:

i. The integral $\int_{a}^{b} f_{k} d g$ exists for each $k \in \mathbb{N}$.

ii. $\lim _{k \rightarrow \infty} f_{k}(x)=f(x)$ for all $x \in[a, b]$,

iii. There exist $\beta, \gamma \in \mathbb{R}$ such that the inequalities

$$
\beta \leq \sum_{i=1}^{n} \int_{u_{(i-1)_{n}}}^{u_{i_{n}}} f_{s_{i}} d g \leq \gamma,
$$

holds for all partitions $P_{n}$ of $[a, b]$ and all $s_{1}, s_{2}, \cdots, s_{n} \in \mathbb{N}$. Then, $f_{k}$ is uniformly Sequential Henstock Stieltjes integrable with respect to $g$. Then, the integral $\int_{a}^{b} f d g$ exists and

$$
\lim _{k \rightarrow \infty} \int_{a}^{b} f_{k} d g=\int_{a}^{b} f d g
$$

Moreover, we have

$$
\lim _{k \rightarrow \infty}\left(\sup _{t \in[a, b]}\left|\int_{a}^{t} f_{k} d g-\int_{a}^{t} f d g\right|\right)=0
$$

\section{$2 \quad$ Main Results}

We state and give the proof of theorems in our main results.

Theorem 2.1. (Dominated Convergence). Let $f_{k}:[a, b] \rightarrow \mathbb{R}$ be a sequence of functions which is Sequential Henstock Stieltjes integrable with respect to an increasing function $g:[a, b] \rightarrow \mathbb{R}$ and is satisfying the following conditions:

i. The integral $\int_{a}^{b} f_{k} d g$ exists for each $k \in \mathbb{N}$,

ii. $\lim _{k \rightarrow \infty} f_{k}(x)=f(x)$ for all $x \in[a, b]$,

iii. There exist Sequential Henstock Stieltjes integrable functions $h_{i}, h_{2}:[a, b] \rightarrow \mathbb{R}$ such that $\int_{a}^{b} h_{1} d g$ and $\int_{a}^{b} h_{2} d g$ exist, where $h_{1} \leq f_{k} \leq h_{2}$ on $[a, b]$ for each $k \in \mathbb{N}$. 
Then, $f_{k}$ is uniformly Sequential Henstock Stieltjes integrable with respect to $g$. Then, the integral $\int_{a}^{b} f d g$ exists and

$$
\lim _{k \rightarrow \infty} \int_{a}^{b} f_{k} d g=\int_{a}^{b} f d g
$$

Moreover, we have

$$
\lim _{k \rightarrow \infty}\left(\sup _{t \in[a, b]}\left|\int_{a}^{t} f_{k} d g-\int_{a}^{t} f d g\right|\right)=0
$$

Proof. From Lemma 1.1, following from condition (iii). Let $\beta=U\left(h_{1}, d g, P_{n}\right)$ and $\gamma=U\left(h_{2}, d g, P_{n}\right)$.

If $P_{n}$ is a sequence of divisions on $[a, b]$ and $s_{1}, s_{2}, \cdots, s_{n} \in \mathbb{N}$. i.e.

$P_{n}=\left(u_{(i-1)_{n}}, u_{i_{n}}\right) \in[a, b]$ for $n=1,2,3, \cdots$, then

$$
\beta=U\left(h_{1}, d g, P_{n}\right) \leq \sum_{i=1}^{n} \int_{u_{(i-1)_{n}}}^{u_{i_{n}}} f_{s_{i}} d g \leq U\left(h_{2}, d g, P_{n}\right)=\gamma .
$$

This shows that the assumption of Lemma 1.1 is satisfied and the proof is complete.

Theorem 2.2.(Bounded Convergence). Let $f_{n}:[a, b] \rightarrow \mathbb{R}$ be a sequence of function and $g:[a, b] \rightarrow \mathbb{R}$ be an increasing function satisfying the following conditions:

i. The integral $\int_{a}^{b} f_{k} d g$ exists for each $k \in \mathbb{N}$

ii. $\lim _{k \rightarrow \infty} f_{k}(x)=f(x)$ for all $x \in[a, b]$,

iii. There exist a constant $M \geq 0$ such that $\left|f_{k}(x)\right| \leq M$ for all $k \in \mathbb{N}$ and $x \in[a, b]$. Then, $f_{k}$ is uniformly Sequential Henstock Stieltjes integrable with respect to $g$, the integral $\int_{a}^{b} f d g$ exists and

$$
\lim _{k \rightarrow \infty} \int_{a}^{b} f_{k} d g=\int_{a}^{b} f d g
$$

Moreover, we have

$$
\lim _{k \rightarrow \infty} \sup _{t \in[a, b]}\left(\left|\int_{a}^{t} f_{n} d g-\int_{a}^{t} f d g\right|\right)=0 .
$$

Proof. If $P_{n}$ is a sequence of partitions on $[a, b]$ and $s_{1}, s_{2}, \cdots, s_{n} \in \mathbb{N}$. where $P_{n}=\left(u_{(i-1)_{n}}, u_{i_{n}}\right) \in[a, b]$ for $n=1,2,3, \cdots$, then

$$
\left|\sum_{i=1}^{n} \int_{u_{(i-1) n}}^{u_{i_{n}}} f_{s_{i}} d g\right| \leq \sum_{i=1}^{n}\left|\int_{u_{(i-1) n}}^{u_{i_{n}}} f_{s_{i}} d g\right| \leq \sum_{i=1}^{n} \operatorname{Mvar}_{u(i-1)_{n}}^{u_{i_{n}}} g=\operatorname{Mvar}_{a}^{b} g .
$$

by the assumptions of Lemma 1.1, which is also satisfied with

$$
-\operatorname{Mvar}_{a}^{b} g \leq\left|\sum_{i=1}^{n} \int_{u_{(i-1) n}}^{u_{i_{n}}} f_{k} d g\right| \leq \operatorname{Mvar}_{a}^{b} g .
$$

This completes the proof.

\section{References}

[1] Abbot, S., 2001, Understanding analysis, Springer Science and Business Media, New York, 200-250.

[2] Bartle, R., 2000, Modern theory of integration, graduate studies in mathematics, American Mathematical Society, 32, Providence RL, 12-40.

[3] Gordon, R., 1994, The Integral of Lebesgues, Denjoy, Perron and Henstock, Graduate studies in Mathematics. American Mathematical Society, 4, Providence RI, 121-150. 
[4] Hamid, M. E, Xu, L. and Gong, Z., 2017, The Henstock-Stieltjes integral for set valued functions, Inter. J. Pure Appl. Math., 114(2), 261-275.

[5] Laramie, P. A., 2016, Sequential approach to the Henstock integral, Washington State University, arXiv:1609.05454v1 [maths.CA], 3-5.

[6] Supriya, D., Lee, Y. and Ganguli, D. A., 2008, Generalised Henstock Stieltjes integral in division functions, Math. Slovaca., 58(4), 653-660. 